This item is the archived peer-reviewed author-version of:

Coping strategy influences quality of life in patients with advanced lung cancer by mediating mood

\title{
Reference:
}

van de Wiel Mick, Derijcke Sofie, Galdermans Daniella, Daenen Marc, Surmont Veerle, De Droogh Els, Lefebure Anneke, Saenen Erika, Vandenbroucke Elke,

Morel Ann-Marie, ....- Coping strategy influences quality of life in patients with advanced lung cancer by mediating mood

Clinical lung cancer - ISSN 1525-7304 - 22:2(2021), p. 146-152

Full text (Publisher's DOI): https://doi.org/10.1016/J.CLLC.2020.09.010

To cite this reference: https://hdl.handle.net/10067/1783080151162165141 


\section{Coping strategy influences quality of Life in patients with advanced lung cancer by mediating mood}

van de Wiel $M^{1}$, Derijcke $S^{2}$, Galdermans $D^{3}$, Daenen $M^{4}$, Surmont $V^{5}$, De Droogh $E^{3}$, Lefebure $A^{6}$, Saenen $E^{7}$, Vandenbroucke $E^{8}$, Morel $A M^{9}$, Sadowska $A^{10}$, van Meerbeeck JP ${ }^{1}$, Janssens $A^{1}$

Corresponding author: van de Wiel Mick,

${ }^{1}$ University Hospital Antwerp, Department of Thoracic Oncology, Wilrijkstraat 10, 2650 Edegem, Belgium

e-mail address: mick.vandewiel@uza.be

Tel: +3238214580

Fax: +3238214102

${ }^{2}$ Department of Pulmonology-Thoracic Oncology, AZ Groeninge, Kortrijk, Belgium ${ }^{3}$ Department of Pulmonology-Thoracic Oncology, ZNA Middelheim, Antwerp, Belgium ${ }^{4}$ Department of Pulmonology, Ziekenhuis Oost Limburg, Genk, Belgium ${ }^{5}$ Pulmonology-Thoracic Oncology Ghent University, Ghent, Belgium ${ }^{6}$ Department of Pulmonology-Thoracic Oncology, ZNA STER, Antwerp, Belgium ${ }^{7}$ Lung diseases/Allergology, AZ Heilige Familie, Reet, Belgium ${ }^{8}$ Department of Pulmonology-Thoracic Oncology, AZ Monica, Antwerp, Belgium ${ }^{9}$ Department of Pulmonology, Sint-Jozefkliniek Bornem \& Willebroek, Bornem, Belgium ${ }^{10}$ Department of Pulmonology, Ziekenhuis Maas en Kempen, campus Maaseik, Maaseik, Belgium

KeyWords: lung cancer, early palliative care, communication, quality of life, coping strategies

\section{Abstract}

\section{Introduction}

Patients with advanced lung cancer experience high physical symptom burden with substantial psychological distress. Depressive and anxiety symptoms are common and associated with worse Quality of Life (QoL). Early palliative care (EPC) addresses the complex supportive care needs improving QoL and mood. The mechanisms of EPC are uncertain. We examined whether and how coping strategy, a primary component of early palliative care, influenced QoL in these patients. 


\section{Materials and Methods}

We conducted a multi-centre cross-sectional study of patients with advanced lung cancer. 125 patients completed assessments of QoL (QLQ-C15-PAL), depressive and anxiety symptoms (HADS) and coping (brief COPE questionnaire). The data were analysed by descriptive statistics. To determine whether and how coping strategy influences QoL, correlations and logistic regressions were performed.

\section{Results}

Positive reframing correlates significantly with global QoL $(r=.25, p<.01)$, emotional well-being $(r=.33, p<.01)$, pain $(r=-.30, p<.01)$, fatigue $(r=-.22, \mathrm{p}$ $<.01)$, loss of appetite $(r=-.22, p<.01)$ and nausea $(r=-.24, p<.01)$. Selfblame correlates significantly with worse emotional well-being $(r=-.19, p<$ $.05)$ and insomnia $(r=.19, p<.05)$. Using a 4 step logistic regression model, it was found that anxiety and depressive symptoms fully mediated the relationship between positive reframing and quality of life.

\section{Conclusion}

Patients with advanced lung cancer using positive reframing as coping strategy, experience higher QoL. The mechanism behind it seems that positive reframing goes along with less anxiety and depressive symptoms leading to a better QoL. Self-blame leads to more insomnia and worse emotional wellbeing. Providing skills to cope effectively could impact QoL in these patients.

\section{Introduction}

Advanced lung cancer is associated with a poor prognosis and a median survival time of approximately ten months.[1] From the moment of diagnosis patients experience variety of concerns. Not only do these patients have to deal with a high burden of symptoms and poor quality of life, they also have to encounter a variety of difficult medical decisions regarding their cancer therapy with palliative intent. Patients have to decide if the possible benefits of cancer treatment outweigh the possible significant toxicities and unwanted side effects. Quality of life is key in the treatment of patients with advanced lung cancer.

It is known that mood influences quality of life and prognosis [2], but it remains uncertain whether and how coping strategies also play a role in this relationship. Early palliative care leads to significant improvements in quality of life, mood and even survival in metastatic lung cancer patients.[3]

It has been suggested that by adding psychological support to early palliative care, enhancing favourable coping strategies, could have a positive effect on quality of life in patients with anxious or depressive symptoms. $[4,5]$ This study 
is conducted to identify these possible coping strategies that could influence quality of life in patients with anxiety or depressive symptoms.

\section{Materials and Methods}

\section{Centres and patients}

This multi-centre cross-sectional study was conducted in 10 hospitals throughout Flanders, Belgium: Antwerp University Hospital, AZ Groeninge Kortrijk, ZNA Sint-Erasmus Borgerhout, AZ Monica Deurne, Sint-Jozef Kliniek Bornem \& Willebroek, Ghent University Hospital, ZNA Middelheim Antwerp, Heilige Familie Reet, Ziekenhuis Oost Limburg and Ziekenhuis Maas en Kempen, Maaseik. All ethical committees of the corresponding hospitals approved the study.

Patients with advanced lung cancer were eligible for inclusion in the study if they were older than 18 and were able to read and write in Dutch. After obtaining a written informed consent, the treating physician asked eligible patients to complete questionnaires, either when visiting the outpatient clinic or when hospitalized. The following data were obtained: age, gender, marital status, religion and WHO performance status. $[6,7]$ Other results of this study, regarding prognostic understanding, are published separately.[8]

\section{Questionnaires}

Quality of Life was evaluated using the QLQ-C15-PAL, a shortened version of the EORTC QLQ-C30, which is a widely used instrument for measuring QoL in cancer research.[9] The QLQ-C15-PAL questionnaire contains 15 questions that cover the 10 most frequent issues, identified as very important, in the palliative population. The tool consists of two multi-item functional scales (physical and emotional functioning), two multi-item symptom scales (fatigue and pain), five single item symptom scales (nausea/vomiting, dyspnoea, insomnia, appetite loss and constipation) and one final overall QoL scale. All items are rated from 1 (not at all) to 4 (very much) in severity, except for the overall QoL scale, which is rated from 1 (very poor) to 7 (excellent). A high score on the symptom scale suggests increased distress, whereas a high score on the functional scale shows increased functional ability. For each item, each scale is converted to a score ranging from 0 to 100 .

Mood (depression and anxiety) was measured using the Hospital Anxiety and Depression Scale (HADS), which consist of 14 items: 7 items on the anxiety subscale (HADS Anxiety) and 7 on the depression subscale (HADS depression). HADS Anxiety focuses mainly on the symptoms of generalized anxiety disorder 
and HADS Depression focuses on anhedonia, the main symptom of depression.[10] Each item is scored on a response scale with four alternatives, ranging from 0 to 3 . After adjusting for six items that are reverse scored, all responses are summed to obtain the two subscales. Recommended cut-off scores according to Zigmond \& Snaith [11] are 8-10 for doubtful cases and >= 11 for definite cases. An optimal balance between sensitivity and specificity has been found using a cut-off score of 8 for both HADS Anxiety and HADS Depression.[12]

Coping strategy was assessed using the brief COPE questionnaire.[13, 14] We incorporated six coping strategies that are considered highly relevant to patients with incurable cancer: emotional support, positive reframing, active coping, acceptance, self-blame and denial.[15, 16] The developer of the instrument encouraged such selective and flexible use of subscales. Participants select the degree to which they have used each type of coping ( $1=$ not at all, 2 = a little bit, $3=$ a medium amount and $4=$ a lot).

\section{Data analysis}

All data were collected and encoded in an SPSS database (IBM, SPSS statistics, version 24 ) by the principal investigator. A sum score was calculated for all scales with a median, standard deviation and Cronbach's $\alpha$. The data were analysed using descriptive statistics. Pearson correlation coefficient or regression analysis and multiple hierarchical regression analysis were used to determine the relationships between different parameters. The results were considered significant at $p=0.05$.

\section{Results}

The trial was conducted between the 1th of May and the 31th of June 2017. In total, 128 patients from 10 hospitals were asked to participate in this study. 3 patients were not willing to fill out the questionnaire: 1 was too tired, 1 reported being tired of filling out questionnaires and 1 did not give a reason. Ultimately, 125 patients were included in the trial. Table 1 shows the sociodemographic characteristics of all patients. [8]

\begin{tabular}{lll}
\hline Age, median (range) & & $65(41-85)$ \\
\hline \multirow{2}{*}{ Gender (\%) } & Male & $75(60 \%)$ \\
& Female & $50(40 \%)$ \\
\hline \multirow{2}{*}{ WHO status, grade (\%) } & 0 & $23(19 \%)$ \\
& 1 & $47(56 \%)$ \\
& 2 & $35(28 \%)$ \\
& 3 & $18(15 \%)$ \\
\hline
\end{tabular}




\begin{tabular}{lll}
\hline & Married & $85(69 \%)$ \\
Marital Status (\%) & Unmarried & $11(9 \%)$ \\
& Divorced & $18(15 \%)$ \\
& Widow(er) & $10(8 \%)$ \\
\hline \multirow{2}{*}{ Religious (\%) } & Yes & $65(59 \%)$ \\
& No & $45(41 \%)$ \\
\hline \multirow{2}{*}{ Children (\%) } & Yes & $113(91 \%)$ \\
& No & $11(9 \%)$ \\
\hline Table 1: demographic characteristics (total patients $n=125)$ \\
\hline
\end{tabular}

Table 2 shows the range, number of items, mean, standard deviation (SD) and Cronbach's $\alpha$ of mood, coping strategy and the different dimensions of quality of life. Acceptance and emotional support have the highest mean scores, while denial and self-blame have the lowest. Emotional support is most used in this patient population with a mean score of 4 . When looking at the frequency of emotional support, only 10 patients claim not using this as a coping strategy. Because of this distribution, we won't use this coping strategy for further analysis because there is no meaningful difference in this patient population. It seems they almost all use emotional coping. (Table 3 )

\begin{tabular}{|c|c|c|c|c|c|}
\hline & Range & $\begin{array}{l}\text { Number } \\
\text { of items }\end{array}$ & Mean & $\begin{array}{l}\text { Standard } \\
\text { Deviation }\end{array}$ & $\begin{array}{c}\text { Cronbach's } \\
\alpha\end{array}$ \\
\hline \multicolumn{6}{|l|}{ Mood } \\
\hline Anxiety & $0-21$ & 7 & 6.5 & 4.1 & .81 \\
\hline Depression & $0-18$ & 7 & 6.4 & 3.6 & .72 \\
\hline \multicolumn{6}{|l|}{ Coping Strategies } \\
\hline Acceptance & $1-4$ & 2 & 3.0 & .75 & .69 \\
\hline Denial & $1-4$ & 2 & 1.8 & .89 & .78 \\
\hline Active Coping & $1-4$ & 2 & 2.3 & .60 & .81 \\
\hline Emotional support & $1-4$ & 2 & 3.5 & .66 & .89 \\
\hline Positive reframing & $1-4$ & 2 & 2.5 & .84 & .69 \\
\hline Self-blame & $1-4$ & 2 & 1.7 & .85 & .79 \\
\hline \multicolumn{6}{|l|}{ Quality of Life (QoL) } \\
\hline General QoL & $0-100$ & 1 & 61.29 & 19.4 & NA \\
\hline Physical well-being & $0-100$ & 3 & 78.6 & 22.34 & .70 \\
\hline Emotional well-being & $0-100$ & 2 & 72.4 & 24.87 & .73 \\
\hline Pain & $0-100$ & 2 & 26.37 & 30.06 & .87 \\
\hline Fatigue & $0-100$ & 2 & 24.6 & 27.44 & .77 \\
\hline Dyspnoea & $0-100$ & 1 & 32.5 & 31.66 & NA \\
\hline Insomnia & $0-100$ & 1 & 33.3 & 34.93 & NA \\
\hline Loss of appetite & $0-100$ & 1 & 31.4 & 35.4 & NA \\
\hline Constipation & $0-100$ & 1 & 14.6 & 27.7 & NA \\
\hline Nausea & $0-100$ & 1 & 18.0 & 28.47 & NA \\
\hline
\end{tabular}


Table 2: range, number of items, mean, standard deviation and Cronbach's $\alpha$ of mood, coping strategies and the different dimensions of quality of life.

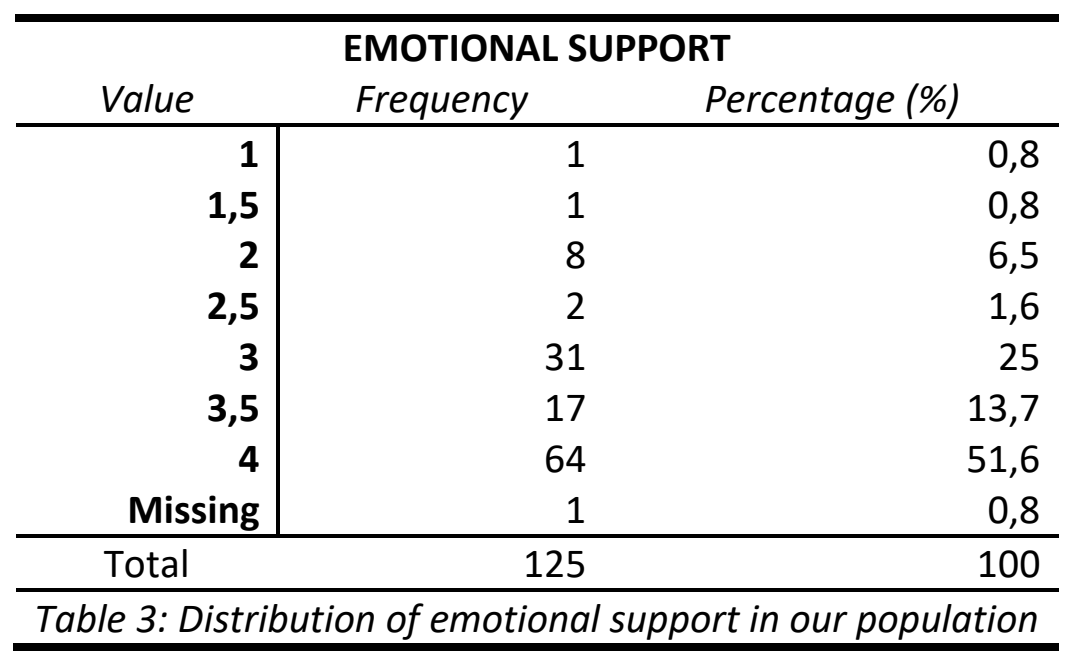

In table 4 , the percentages of patients suffering anxiety or depression are shown. $20 \%$ and $26 \%$ of the patients had mild complaints related to anxiety or depression, respectively; $11 \%$ and $10 \%$ had moderate complaints and $4 \%$ and $2 \%$ were severely anxious or severely depressed.[8]

\begin{tabular}{lll}
\hline HADS Scores & Anxiety (\%) & Depression (\%) \\
\hline $0-7$ (normal) & 65 & 62 \\
$8-10$ (mild) & 20 & 26 \\
$11-14$ (moderate) & 11 & 10 \\
$>14$ (severe) & 4 & 2 \\
\hline
\end{tabular}

Table 4: percentage of patients with normal, mild, moderate or severe complaints

First, Table 5 shows the correlations between mood and QoL. Anxiety correlates significantly with worse general QoL $(r=-.23, p=.02)$, worse physical well-being $(r=-.21, p=.03)$, worse emotional well-being $(r=-.73, p=$ $.001)$, more pain $(r=.37, p=.000)$, more dyspnoea $(r=.21, p=.03)$, more insomnia $(r=.38, p=.000)$ and more constipation $(r=.21, p=.03)$.

Depression correlates significantly with worse general QoL $(r=-.63, p=.000)$, worse physical well-being $(r=-.44, p=.000)$, worse emotional well-being $(r=-$ $.44, p=.00)$, more pain $(r=.46, p=.000)$, more fatigue $(r=.49, p=.00)$, more dyspnoea $(r=.44, p=.00)$, more insomnia $(r=.20, p=.04)$, more loss of appetite $(r=.45, p=.000)$, more nausea $(r=.29, p=.002)$ and more constipation $(r=.27, p=.003)$

Table 5 also shows the correlations between coping strategies and QoL. Positive reframing correlates significantly with better overall QoL $(r=.25, p<$ 
$.01)$, better emotional well-being $(r=.33, p<.01)$, less pain $(r=-.30, p<.01)$, less fatigue $(r=-.22, p<.01)$, less loss of appetite $(r=-.22, p<.01)$ and less nausea $(r=-.24, p<.01)$. Self-blame correlates significantly with worse emotional well-being $(r=-.19, p<.05)$ and more insomnia $(r=.19, p<.05)$.

Table 6 shows the correlations between mood and coping strategies. Anxiety correlates with acceptance $(r=-.30, p=.001)$, active coping $(r=.27, p=.004)$, positive reframing $(r=-.22, p=.02)$ and self-blame $(r=.36, p<.000)$. The coping strategies self-blame and active coping reinforce anxiety, whereas acceptance and positive reframing are buffering coping strategies regarding anxiety.

Depression correlates with positive reframing $(r=-.25, p=.007)$, thus working as a buffering coping strategy regarding depression. 


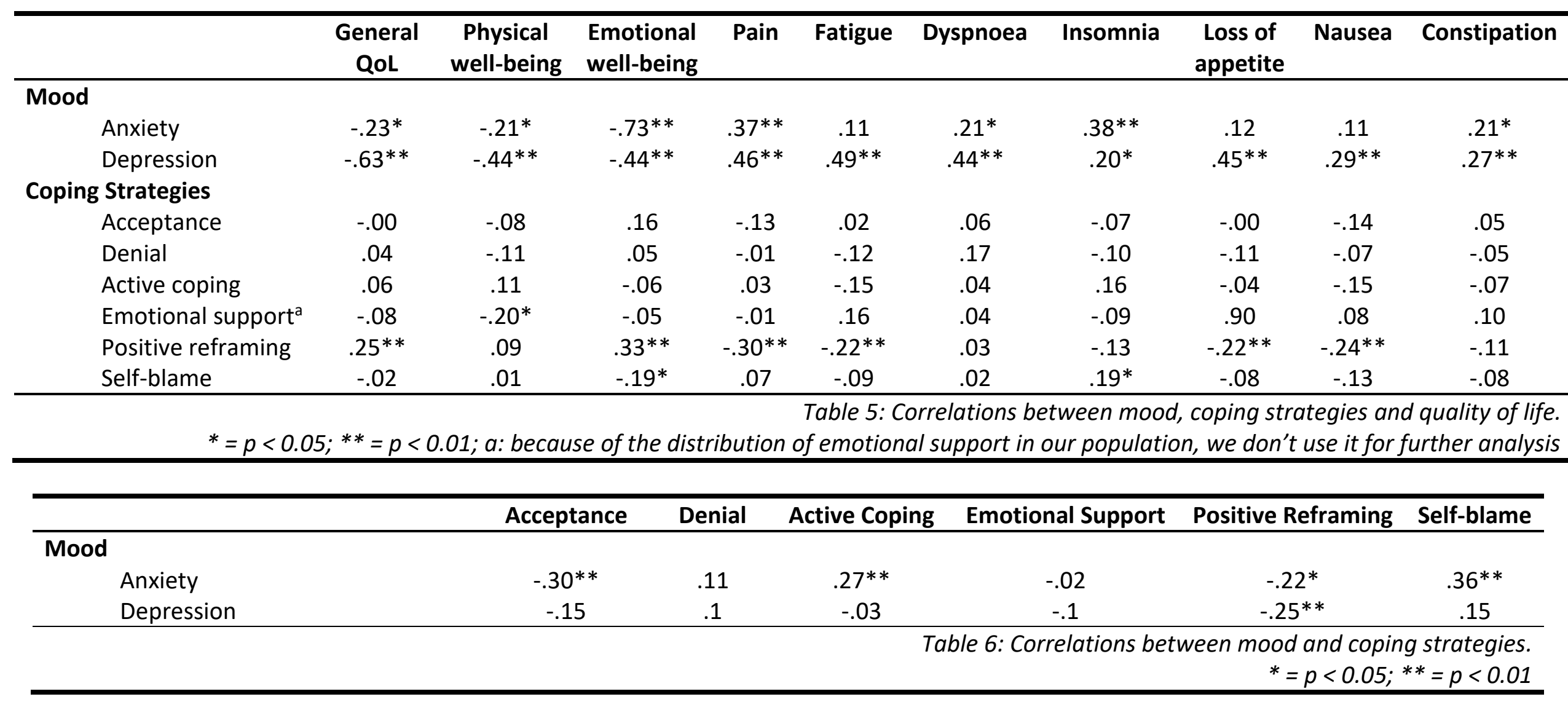


Figure 1 represents the mediation model. In step 1 of the mediation model, the regression of positive reframing on quality of life (QoL), ignoring the mediator, was significant $(\beta=.25, t(121)=2.85, p=<.005)$. Step 2 showed that the regression of positive reframing on the mediator, anxiety, was also significant $(\beta=-.22, t(110)=-2.37, p=<.02)$. Step 3 of the mediation process showed that the mediator (anxiety), controlling for QoL, was significant ( $\beta=$ $.23, \mathrm{t}(110)=-2.48, \mathrm{p}=.015)$. Step 4 of the analyses revealed that, controlling for the mediator (anxiety), positive reframing score was not a significant predictor of $\mathrm{QoL}(\beta=.16, \mathrm{t}(108)=1.78, \mathrm{p}=.08)$. It was found that anxiety fully mediated the relationship between positive reframing and quality of life.

In step 1 of the mediation model, the regression of positive reframing on quality of life (QoL), ignoring the mediator, was significant $(\beta=.25, t(121)=$ $2.85, p=<.005)$. Step 2 showed that the regression of positive reframing on the mediator, depression, was also significant $(\beta=-.25, \mathrm{t}(113)=-2.37, \mathrm{p}=$ $<.007)$. Step 3 of the mediation process showed that the mediator (depression), controlling for QoL, was significant $(\beta=-.63 \mathrm{t}(113)=-8.56, p=$ .00). Step 4 of the analyses revealed that, controlling for the mediator (depression), positive reframing score was not a significant predictor of QoL(b $=.09, \mathrm{t}(111)=1.17, \mathrm{p}=.24)$. It was found that depressive symptoms fully mediated the relationship between positive reframing and quality of life.

Anxiety/ Depressive symptoms

Positive Reframing

Quality of Life

Fig 1: Mediating mood by positive reframing

\section{Discussion}

Consistent with previous studies, our study shows that lung cancer patients have a high psychological symptom burden, with $40 \%$ being anxious and/or having depressive symptoms.[2, 3, 17, 18] Symptoms of depression and anxiety are associated with poorer treatment adherence, increased length of hospital stay, worse QoL, and even survival. Our study also indicates that mood is the most important factor influencing on different aspects of QoL, with a 
worse QoL in patients experiencing anxiety and/or depressive symptoms.[2, 19] Several studies have shown the association between depression and survival in patients with advanced lung cancer. Some studies report a worse survival for patients with depressive symptoms[2, 20-22], while other studies don't report any difference.[23, 24] No association between anxiety and survival in lung cancer patients has been found.[2, 20,21] All these findings indicate that an adequate identification and treatment of both anxious and depressive symptoms in lung cancer patients could lead to an improvement in QoL and maybe survival.

Hsieh and his colleagues conducted a meta-analysis of 12 randomised controlled trials examining the overall effects of supportive care interventions on depressive symptoms in lung cancer patients. Their results indicate that supportive care interventions are able to improve depressive symptoms in lung cancer patients. The most effective types of treatment were psychotherapy combined with psychoeducation and psychotherapy combined with an exercise program. No conclusions regarding effect on QoL or survival have been made.[25] Little is known about the use of anti-depressants in advanced cancer patients. Anti-depressants have the disadvantage of taking a period of time before any improvement in the mood of the patient may occur. They also cause unpleasant side-effects (dry mouth, constipation, ...) which are often already present in cancer patients. No studies regarding the effect of antidepressants on depressive symptoms in cancer patients have been conducted. Randomized studies evaluating both pharmacological and nonpharmacological management on anxiety/depression and their impact on QoL and prognosis are needed.

It has been reported earlier that coping plays an essential role in maintaining the well-being in cancer patients.[15, 16, 26] A recent article reviews evidence supporting the beneficial effects of palliative care on patient coping as well as the mechanisms by which improved coping is associated with better outcomes.[27] Our results also show that coping strategies can positively influence different aspects of Quality of Life, mediated by mood.

A variety of coping strategies have been identified [28-30], with an association between coping strategy and QoL in cancer patients [31], critically ill patients [32] and in patients with heart failure.[33] Patients often initially draw on their existing coping strategies before their cancer diagnosis to manage distress and psychological well-being.[34] In a qualitative study by Link et al. it is shown that physicians play an essential role in the way patients choose a coping 
strategy leading to more favourable coping strategies to deal with their cancer diagnosis.[35] In our study, almost all advanced lung cancer patients use emotional support and active coping as coping strategy, probably because of its effectiveness as shown in previous studies. We could not interpret correlations because there is no meaningful difference between using emotional support 3 or 4 on a scale from 1 to 4 . This is also the case for active coping. Acceptance correlates with less anxiety. Self-blame goes along with worse emotional well-being and more insomnia. Positive reframing on the other hand goes along with better QoL. The mechanism behind it seems the fact that positive reframing leads to better mood (less anxiety and depressive symptoms) conducting better QoL. Nipp et al. studied the relationship between coping strategies, mood and QoL. Likewise with our results, they saw a higher median score for emotional support and acceptance and a lower median score for denial and self-blame in their study population. They found lower depression scores in patients using emotional support and acceptance as a coping strategy, while denial and self-blame correlated with higher depression scores. Regarding anxiety, the use of acceptance coping correlated with lower anxiety scores, while denial and self-blame coping strategies correlated with higher anxiety scores. Consistently, a better QoL was seen in patients who used emotional support and acceptance and a lower QoL was seen in patients using denial and self-blame.[16] In a study by Greer at al. in patients with advanced lung and gastro-intestinal cancer, it was shown that a positive change in patient coping accounted for significant improvement in QoL and depressive symptoms. This positive effect could be shaped by early palliative care interventions.[26, 36] Consistently, in a study by Hoerger et al. coping was the only content area of early palliative care that was associated with improved patient-reported outcomes.[37] Winger et al. found significant effects of coping on quality of life by using Telephone Symptom Management (TSM) as a way to enhance coping skills in cancer patients.[38] Assessing patients' coping can not only help us identify those who are at increased risk of distress, but can also help us guide in the treatment of adapting that specific coping behaviour.[39]

Different studies have showed that teaching coping strategies during an early palliative care is feasible and acceptable in clinical practice.[37, 40, 41] Porter et al. showed that coping skills training led to improvement in a range of outcomes, including quality of life, in lung cancer patients.[42] However in a recent pilot study by Yang and colleagues no benefit was shown on quality of life and mood when teaching coping strategies through their EQUIP intervention. It is possible that this could be due to a low prevalence of 
targeted symptoms, stating that more research in bigger patient groups is needed.[43]

The general limitation of cross-sectional research applies to this study. Patients were surveyed only once, no comparison was made over time and thus causality cannot be evaluated. As a second limitation we mark that only 3 patients were not willing to complete the questionnaire. Patients who chose not to participate may differ a lot from the population used in this study. Lastly, We did not collect information about patients' use of other services (psychiatric counselling, social work,), which may affect patients' coping, QoL and mood. However, because of the fact that the study was conducted early in the progress of lung cancer diagnosis it seems less likely that patients already received these services. Future studies should focus on the longitudinal impact of coping strategies and patients' QoL. We suggest a randomized study where caregivers teach different coping strategies to patients who experience anxiety and/or depressive symptoms in a way to protect these patients against a poor QoL.

\section{Conclusion}

The quality of life in patients with advanced lung cancer is highly influenced by their mood. Approximately $40 \%$ of patients are anxious or have depressive symptoms. Early recognition of these symptoms, patients' coping style and adequate access to psychological support remains a challenge in standard oncology care. Targeting anxiety and or depressive symptoms could lead to better QoL, less hospital admissions and even better survival in lung cancer patients. This could be achieved by teaching adequate coping skills in patients experiencing these symptoms. Patients using positive reframing as coping strategy, experience higher QoL. The mechanism behind it seems that positive reframing goes along with less anxiety and depressive symptoms leading to a better QoL. Early Palliative Care (EPC) could act as a method to teach these coping skills in lung cancer patients.

\section{Conflict of interest}

All authors have nothing to declare.

\section{Funding}

This research did not receive any specific grant from funding agencies in the public, commercial, or not-for-profit sectors. 


\section{References}

1. Pilkington, G., et al., A systematic review of the clinical effectiveness of first-line chemotherapy for adult patients with locally advanced or metastatic non-small cell lung cancer. Thorax, 2015. 70(4): p. 359-67.

2. Arrieta, O., et al., Association of depression and anxiety on quality of life, treatment adherence, and prognosis in patients with advanced non-small cell lung cancer. Ann Surg Oncol, 2013. 20(6): p. 1941-8.

3. Temel, J., et al., Early Palliative Care for Patients with Metastatic Non-Small-Cell Lung Cancer. N Engl J Med, 2010. 363(8): p. 733-742.

4. Czerw, A.I., U. Religioni, and A. Deptala, Adjustment to Life with Lung Cancer. Adv Clin Exp Med, 2016. 25(4): p. 733-40.

5. Yoong, J., et al., Early palliative care in advanced lung cancer: a qualitative study. JAMA Intern Med, 2013. 173(4): p. 283-90.

6. Oken, M.M., et al., Toxicity and response criteria of the Eastern Cooperative Oncology Group. Am J Clin Oncol, 1982. 5(6): p. 649-55.

7. Sorensen, J.B., et al., Performance status assessment in cancer patients. An interobserver variability study. Br J Cancer, 1993. 67(4): p. 773-5.

8. Janssens, A., et al., Prognostic Understanding and Quality of Life in Patients With Advanced Lung Cancer: A Multicenter Study. Clin Lung Cancer, 2019. 20(3): p. e369-e375.

9. Groenvold, M., et al., The development of the EORTC QLQ-C15-PAL: a shortened questionnaire for cancer patients in palliative care. Eur J Cancer, 2006. 42(1): p. 55-64.

10. Snaith, R.P., The Hospital Anxiety And Depression Scale. Health Qual Life Outcomes, 2003. 1: p. 29.

11. Snaith, R.P. and A.S. Zigmond, The hospital anxiety and depression scale. Br Med J (Clin Res Ed), 1986. 292(6516): p. 344.

12. Bjelland, I., et al., The validity of the Hospital Anxiety and Depression Scale: An updated literature review. Journal of Psychosomatic Research, 2002. 52: p. 69-77.

13. Carver, C.S., You want to measure coping but your protocol's too long: consider the brief COPE. Int J Behav Med, 1997. 4(1): p. 92-100.

14. Hagan, T.L., et al., Coping in Patients With Incurable Lung and Gastrointestinal Cancers: A Validation Study of the Brief COPE. J Pain Symptom Manage, 2017. 53(1): p. 131-138.

15. Nipp, R.D., et al., Coping and Prognostic Awareness in Patients With Advanced Cancer. J Clin Oncol, 2017. 35(22): p. 2551-2557.

16. Nipp, R.D., et al., The relationship between coping strategies, quality of life, and mood in patients with incurable cancer. Cancer, 2016. 122(13): p. 2110-6.

17. Shi, Y., et al., Self-reported depression among patients with non-small cell lung cancer. Thorac Cancer, 2015. 6(3): p. 334-7.

18. Delgado-Guay, M., et al., Symptom distress in advanced cancer patients with anxiety and depression in the palliative care setting. Support Care Cancer, 2009. 17(5): p. 573-9.

19. Nipp, R.D., et al., Factors associated with depression and anxiety symptoms in family caregivers of patients with incurable cancer. Ann Oncol, 2016. 27(8): p. 1607-12.

20. Pirl, W.F., et al., Depression after diagnosis of advanced non-small cell lung cancer and survival: a pilot study. Psychosomatics, 2008. 49(3): p. 218-24. 
21. Chen, M.L., M.C. Chen, and C.T. Yu, Depressive symptoms during the first chemotherapy cycle predict mortality in patients with advanced non-small cell lung cancer. Support Care Cancer, 2011. 19(11): p. 1705-11.

22. Pirl, W.F., et al., Depression and survival in metastatic non-small-cell lung cancer: effects of early palliative care. J Clin Oncol, 2012. 30(12): p. 1310-5.

23. Akechi, T., et al., Psychosocial factors and survival after diagnosis of inoperable non-small cell lung cancer. Psychooncology, 2009. 18(1): p. 23-9.

24. Nakaya, N., et al., Negative psychological aspects and survival in lung cancer patients. Psychooncology, 2008. 17(5): p. 466-73.

25. Hsieh, C.C. and F.H. Hsiao, The effects of supportive care interventions on depressive symptoms among patients with lung cancer: A metaanalysis of randomized controlled studies. Palliat Support Care, 2017. 15(6): p. 710-723.

26. Greer, J.A., et al., Role of Patient Coping Strategies in Understanding the Effects of Early Palliative Care on Quality of Life and Mood. J Clin Oncol, 2018. 36(1): p. 5360.

27. Greer, J.A., et al., Understanding and Addressing the Role of Coping in Palliative Care for Patients With Advanced Cancer. J Clin Oncol, 2020. 38(9): p. 915-925.

28. Harrop, E., et al., Managing, making sense of and finding meaning in advanced illness: a qualitative exploration of the coping and wellbeing experiences of patients with lung cancer. Sociol Health Illn, 2017. 39(8): p. 1448-1464.

29. Kuo, T.T. and F.C. Ma, Symptom distresses and coping strategies in patients with non-small cell lung cancer. Cancer Nurs, 2002. 25(4): p. 309-17.

30. Liao, Y.C., et al., Psychological distress and coping strategies among women with incurable lung cancer: a qualitative study. Support Care Cancer, 2018. 26(3): p. 989-996.

31. Henoch, I., et al., The impact of symptoms, coping capacity, and social support on quality of life experience over time in patients with lung cancer. J Pain Symptom Manage, 2007. 34(4): p. 370-9.

32. Fok, S.K., S.Y. Chair, and V. Lopez, Sense of coherence, coping and quality of life following a critical illness. J Adv Nurs, 2005. 49(2): p. 173-81.

33. Ekman, I., B. Fagerberg, and B. Lundman, Health-related quality of life and sense of coherence among elderly patients with severe chronic heart failure in comparison with healthy controls. Heart Lung, 2002. 31(2): p. 94-101.

34. Walshe, C., et al., Coping Well with Advanced Cancer: A Serial Qualitative Interview Study with Patients and Family Carers. PLoS One, 2017. 12(1): p. e0169071.

35. Link, L.B., et al., How do cancer patients choose their coping strategies? A qualitative study. Patient Educ Couns, 2005. 58(1): p. 96-103.

36. Irwin, K.E., et al., Early palliative care and metastatic non-small cell lung cancer: potential mechanisms of prolonged survival. Chron Respir Dis, 2013. 10(1): p. 3547.

37. Hoerger, M., et al., Defining the Elements of Early Palliative Care That Are Associated With Patient-Reported Outcomes and the Delivery of End-of-Life Care. J Clin Oncol, 2018. 36(11): p. 1096-1102.

38. Winger, J.G., et al., Coping Skills Practice and Symptom Change: A Secondary Analysis of a Pilot Telephone Symptom Management Intervention for Lung Cancer Patients and Their Family Caregivers. J Pain Symptom Manage, 2018. 55(5): p. 1341-1349.e4. 
39. Prasertsri, N., et al., Repressive coping style: relationships with depression, pain, and pain coping strategies in lung cancer outpatients. Lung Cancer, 2011. 71(2): p. 235-40.

40. Nipp, R.D., et al., Differential effects of early palliative care based on the age and sex of patients with advanced cancer from a randomized controlled trial. Palliat Med, 2018. 32(4): p. 757-766.

41. Zhuang, $\mathrm{H}_{\text {., }}$ et al., Effect of early palliative care on quality of life in patients with non-small-cell lung cancer. Curr Oncol, 2018. 25(1): p. e54-e58.

42. Porter, L.S., et al., Caregiver-assisted coping skills training for lung cancer: results of a randomized clinical trial. J Pain Symptom Manage, 2011. 41(1): p. 1-13.

43. Yang, G.M., et al., Pilot Randomized Phase II Trial of the Enhancing Quality of Life in Patients (EQUIP) Intervention for Patients With Advanced Lung Cancer. Am J Hosp Palliat Care, 2018. 35(8): p. 1050-1056. 